\title{
Collaborative Welding System using BIM for Robotic Reprogramming and Spatial Augmented Reality
}

\author{
Pedro Tavares ${ }^{\mathrm{a}, \mathrm{c}, *}$, Carlos M. Costa ${ }^{\mathrm{b}, \mathrm{c}}$, Luís Rocha ${ }^{\mathrm{b}}$, Pedro Malaca ${ }^{\mathrm{a}}$, Pedro Costa ${ }^{\mathrm{b}, \mathrm{c}}$, \\ António P. Moreira ${ }^{\mathrm{b}, \mathrm{c}}$, Armando Sousa ${ }^{\mathrm{c}}$, Germano Veiga ${ }^{\mathrm{b}, \mathrm{c}}$ \\ ${ }^{a}$ SARKKIS robotics, Portugal \\ ${ }^{\mathrm{b}}$ Centre for Robotics in Industry and Intelligent Systems at INESC TEC, Portugal \\ ${ }^{\mathrm{c}}$ Faculty of Engineering of the University of Porto, Portugal
}

\section{A R T I C L E IN F O}

\section{Keywords:}

Robotic Welding

BIM

Spatial Augmented Reality

Structural Steel Construction Industry

\begin{abstract}
A B S T R A C T
The optimization of the information flow from the initial design and through the several production stages plays a critical role in ensuring product quality while also reducing the manufacturing costs. As such, in this article we present a cooperative welding cell for structural steel fabrication that is capable of leveraging the Building Information Modeling (BIM) standards to automatically orchestrate the necessary tasks to be allocated to a human operator and a welding robot moving on a linear track. We propose a spatial augmented reality system that projects alignment information into the environment for helping the operator tack weld the beam attachments that will be later on seam welded by the industrial robot. This way we ensure maximum flexibility during the beam assembly stage while also improving the overall productivity and product quality since the operator no longer needs to rely on error prone measurement procedures and he receives his tasks through an immersive interface, relieving him from the burden of analyzing complex manufacturing design specifications. Moreover, no expert robotics knowledge is required to operate our welding cell because all the necessary information is extracted from the Industry Foundation Classes (IFC), namely the CAD models and welding sections, allowing our 3D beam perception systems to correct placement errors or beam bending, which coupled with our motion planning and welding pose optimization system ensures that the robot performs its tasks without collisions and as efficiently as possible while maximizing the welding quality.
\end{abstract}

\section{Introduction}

The increasing competitiveness drives the Architecture, Engineering, Construction (AEC) industry to push automated data transfer among design and production stages. In that regard, it is possible to identify key areas that still require standardization and flexibility in order to match the industrial requirements with the development of software and management available to be used in the shop floor of manufacturing industries.

Over the last few years, we have been noticing a clear effort towards improving such conditions while assuring the interoperability between all key processes and partners required to perform a given task. These efforts lead to the creation of an ISO standard for data modeling - the Industry Foundation Classes (IFC) developed by buildingSMART [1]. Up until recently, the IFC based work flows are more commonly seen in the design phase of the building projects, being less available for the fabricators. Despite being a data modeling strategy not completely exploited yet, it shows some interesting characteristics as it allows the creation of an automated information flow between design and production. Furthermore, the IFC can describe the complete design and production process in a unique file, simplifying the integration between teams and companies.

Another result reached by this effort is related to the insertion of automated solutions for solving problems concerning harsh, dangerous and high precision tasks. In particular, robotic solutions excel in reaching these requirements. As such, lately it is possible to clearly identify a tendency for the usage of these solutions $[2,3]$. The goal is to work towards the development of robots that are able to autonomously adapt to several applications and environments. For this to happen robots must become highly efficient operators, able to complete highly

\footnotetext{
* Corresponding author at: SARKKIS robotics, Portugal

E-mail addresses: pedro.tavares@sarkkis.com (P. Tavares), carlos.m.costa@inesctec.pt (C.M. Costa), luis.f.rocha@inesctec.pt (L. Rocha), pedro.malaca@sarkkis.com (P. Malaca), pedro.g.costa@inesctec.pt (P. Costa), antonio.p.moreira@inesctec.pt (A.P. Moreira), asousa@fe.up.pt (A. Sousa), germano.veiga@inesctec.pt (G. Veiga).
} 
demanding tasks and to cooperate with both external machinery and human operators. All these conditions arise in importance when considering the current organizational growth. Currently such growth is associated with the development of small and medium enterprises (SMEs) [4]. Facing this, well structured data models as well as flexible and easily adaptable robotic solution can improve many industrial fields, in particular the steel construction industry regarding the cutting and welding tasks.

Considering the specific use cases of the steel fabricators domain, over the years, the evolution in design methodologies and tools has lead to the usage of computer assisted methods (CAD and CAM). Commonly the result of these systems will guide to the planning and execution of a proposed task.

However, the previous standards, such as DSTV files, were struggling to fulfill the goals, preventing the steel constructing industry from achieving the so desired efficient flow of information, between the design and specification phases and the production stages. Due to these limitations, the steel fabrication sector required the development of a new format with higher acceptance. In that regard, an extension to the IFC standard was considered by major corporations as possibility to close the gap previously identified and fulfill the industrial requirements [5].

Currently, the steel fabrication industry is also demanding the automation of several inherent production processes, as a way to shorten the project's life cycles and reduce the related costs. Several applications can be found actively working in industrial shop floors. These solutions vary from automatic robotic cells for welding and surface treatment and automatic handling [6]. Such advanced robotic systems become effective as a standalone partner in construction.

In this paper we discuss the introduction of this new file format and the main advantages that it can bring to the industrial process, focusing not only on the information flow in the fabrication process, but also in the amount of information that IFC can transfer and how this information contributes for process automation. Furthermore, an efficient automated robotic welding solution will be presented considering several key modules that contribute to the information flow from design specifications to robotic execution, namely Spatial Augmented Reality (SAR) for helping the operator tack weld structures, 3D beam perception for improving robotic seam welding operations along with planning of the robots movements trajectories and task optimization.

The paper is organized as follows. Section 2 presents a brief overview on the current state of development of robotic applications. Section 3 introduces the overall system architecture and provides a description of the key features of the collaborative robotic welding cell. Section 4 presents some experimental results for showing the full integration of the main modules described in previous sections. Finally, Section 5 highlights the overall conclusions and discusses challenges for tackling in the future.

\section{Related Work}

The steel fabrication industry has been using file formats with limited information to manage the production cycle. These limitations have great impact in how the information flows at the shop floor level, increasing the probability of human error and affecting the quality of the products produced by the company.

The Building Information Modeling (BIM) term was first introduce in 1992 [7] and refers to a building design methodology characterized by the generation and use of coordinated and internally consistent computable information about a building project in design and construction.

Despite of the high added value related to the standardization of information throughout all stages of the steel fabrication process, a correct definition of such standard is still debatable. Over the years, the DSTV file was the main file format used in the production process, but the limitations in both geometrical descriptions and additional information to vertically connect design and production stages, raises the need for a more complete file format.

The Industry Foundation Classes (IFC) data model is a file format specification that intends to unite both ends of the process and facilitate interoperability in the architecture, engineering and construction (AEC) industry. The IFC model specification is open, available and registered by ISO as an official International Standard ISO 16739:2013. It is an object-based file format with a data model developed by buildingSMART (formerly the International Alliance for Interoperability, IAI) and is a commonly used collaboration format in Building Information Modeling (BIM) based projects [8].

Lately, the usage of robotic solutions in industrial scenarios has also raised in importance. These kind of solutions ensure process efficiency in a wide range of industrial applications, namely in harsh conditions and for automating repetitive tasks. However, there are some pitfalls concerning their usage in dynamic work cells or complex tasks. In that regards, some efforts have been made in order to provide a more complete and robust approach to these concerns. The usage of sensing systems arise in importance as they allow to generate a correct virtual model describing the industrial environment conditions. One of the main focus of the robotic community is towards building the world model from the perception system. Multiple application have been implemented and validated using laser technology such as laser-dot or laser-line which provides the correct set of detailed information to described parts for object recognition and position detection [9].

Recently, there has been additional efforts regarding human machine interfaces and collaboration between human operators and robotic systems. An official International Standard ISO/TS 15066:2016 [10] has been defined in order to accommodate this ever growing interest of having both robotic systems and human operators working towards the completion of a task. In this domain, SAR systems [11] have been emerging as an intuitive and reliable approach to transmit information to the operator for helping him perform his tasks faster and with less mistakes. By accurately projecting alignment information directly into the environment [12] the operator no longer needs to use error prone and time consuming measuring tools. Moreover, this information can be complemented with wearable devices such as Head Mounted Displays (HMDs) [13-15], smart watches [16,17] and handheld displays [18] for not being restricted by the environment surfaces that act as projection canvas. Besides welding applications, SAR systems using projection mapping techniques have been expanding to other industries, namely in assembly and maintenance tasks in the automotive sector [19] and also in painting shops in aeronautic factories [20].

Robotic applications currently lack in flexibility to autonomously adapt to dynamic constraints that can be found in industrial scenarios. Steel fabrication industries have made efforts towards modularity, flexibility and efficiency improvement of automated robotic solutions mainly in cutting and welding operations [21,22]. Although all the presented works have been validated by the scientific community, they have some limitations when applied on industrial use cases. An integrated solution that uses a complete information flow allied to a flexible human-robot collaboration can be very valuable as it enhances the efficiency of both manual (human) and automated (robot) processes, resulting in a increase in the overall productivity.

\section{System Architecture}

For improving the flexibility and ensuring the long term maintenance of a system, it must follow a modular and scalable architecture for allowing its evolution over time for dealing well with a range of similar tasks. Considering these core characteristics and focusing on a welding robotic system, it is crucial to take into account three major areas, such as, information acquisition and processing, work cell sensing along with system control and adaptation. These can be arranged within the diagram shown in Fig. 1 and will be described in the next 


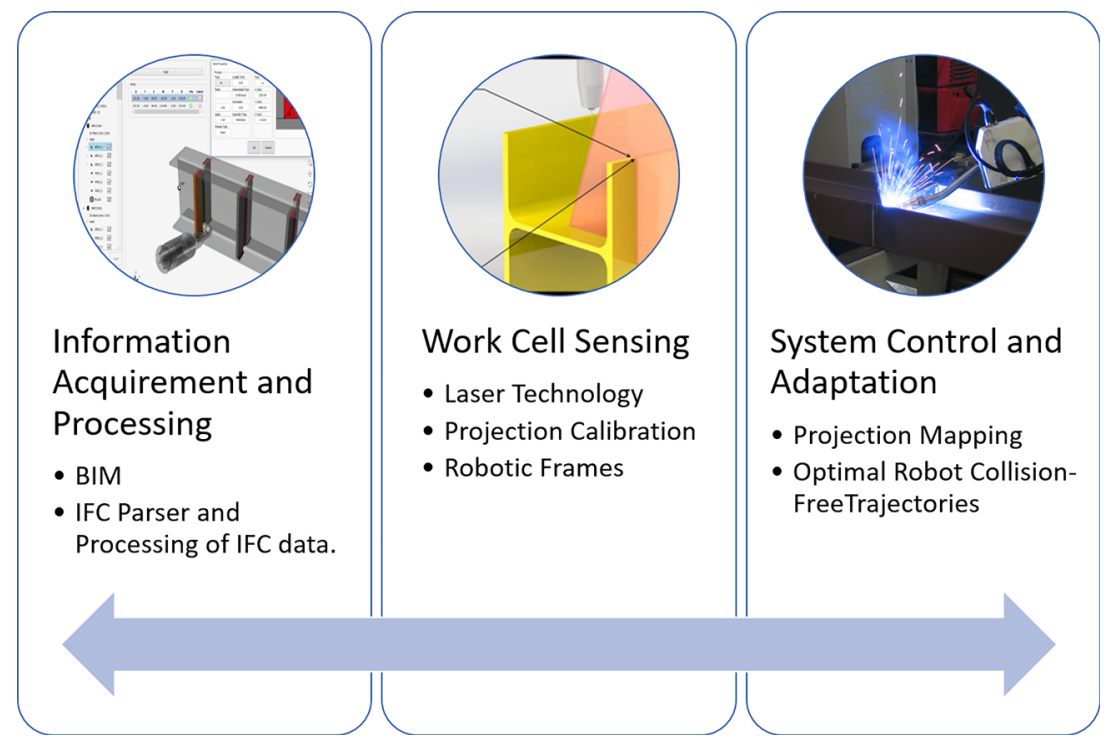

Fig. 1. Architectural breakdown of the design and production.

sections.

The main stages of the proposed work cell are summarized in Fig. 2. It presents how the information extracted from BIM files is used for implementing a collaborative welding cell that includes a human machine interaction system using spatial augmented reality for speeding up the assembly of the beam attachments along with 3D sensing systems for improving the welding quality and a collision free path planner for ensuring safe operation of the welding robot.

\subsection{IFC creation}

Throughout this project, the Tekla software was used for handling CAD and BIM information. Tekla Structures (TS) is the building information modelling software (3D detailing) that has been the reference source of BIM information from which *.ifc format files are obtained and used for production. It was chosen due to its widespread usage in the construction industry (specially steel construction).

The steps to obtain, from TS, the ifc file for a given structure / assembly are fairly straight forward and use Tekla's own IFC export system. Nevertheless some small steps need to be in place prior to export so that all important information is obtained. These sequence are done by activating Tekla's functions in order to properly export a set of crucial welding parameters.

The output is be a IFC file containing all relevant information for the project execution that then is made available for production. Once again, this is a specific approach to Tekla Structures IFC export procedure, known at the moment, expecting that similar BIM modelling applications will generate similar data output.

\subsection{IFC Parser}

Parsing IFC files is not an easy task to accomplish given its complexity and flexibility. The buildingSMART [1] organization provides all the tools and information required to do it, but the complex definition of the elements, the hierarchy and the relation of each other inside the file make this a complex task.

Currently there is an extensive offer of alternatives, free or commercial parsers like the OpenBIM [23] or some of the toolboxes of parsers that can be found on buildingSMART website or IFCWiki [24] for example. This can be a boost for the integration process but will not be enough for software development, requiring some knowledge of the IFC hierarchy to know were you can find in the file the properties that you need for the software development.

\subsection{IFC Information Processing}

Once the IFC data is parsed and transformed into usable information, it is given to the MetroID BeamWeld software (developed by SARKKIS - Robotics) for generating vectors of interest for performing a IFC defined weld operation. The automatic parsing and transformation of IFC data into usable information for programming a welding robot is a huge improvement in the fabrication process, not only for offline programming but also for the traceability of the process (dimensions, materials used, finishes) due to no longer being necessary to be manually done by an operator.

Looking into to the MetroID BeamWeld software, the weld information can dramatically speed up the preparation of the welding operations, since it is no longer necessary to manually detect the joints to be welded within the Computer Aided Design (CAD) models. Using the IFC CAD data and weld specification it is possible to automatically generate the welding vectors shown in Fig. 3. For achieving this, advanced algorithms were developed for detecting the joint line and ensure collision avoidance between the welding torch and the parts to be welded.

\subsection{Spatial Augmented Reality Human-Machine Interface}

Spatial Augmented Reality (SAR) systems using projection mapping techniques can provide immersive Human-Machine Interfaces (HMIs) for helping human operators perform their tasks faster, more accurately and with less mistakes. They have a wide range of applications in the manufacturing industry and when integrated with Building Information Modeling (BIM) systems they can be used for providing contextual information directly into the environment location where it is needed. For our particular use cases, the SAR system that we developed projects into a structural beam the place in which the operator should place and tack weld the metal parts (that will be later on seam welded by a robot). This immersive approach of transmitting the design specifications to the operator allows him to perform the assembly tasks faster and without relying on error prone procedures (such as measuring tapes, ruler squares or protractors). Moreover, given that the information is provided on demand, our system transfers the burden of constantly checking the design schematics from the operator to an automated system, which results in an overall improvement of productivity while reducing possible mistakes when interpreting the manufacturing design specifications.

For achieving a projection accuracy within the manufacturing 

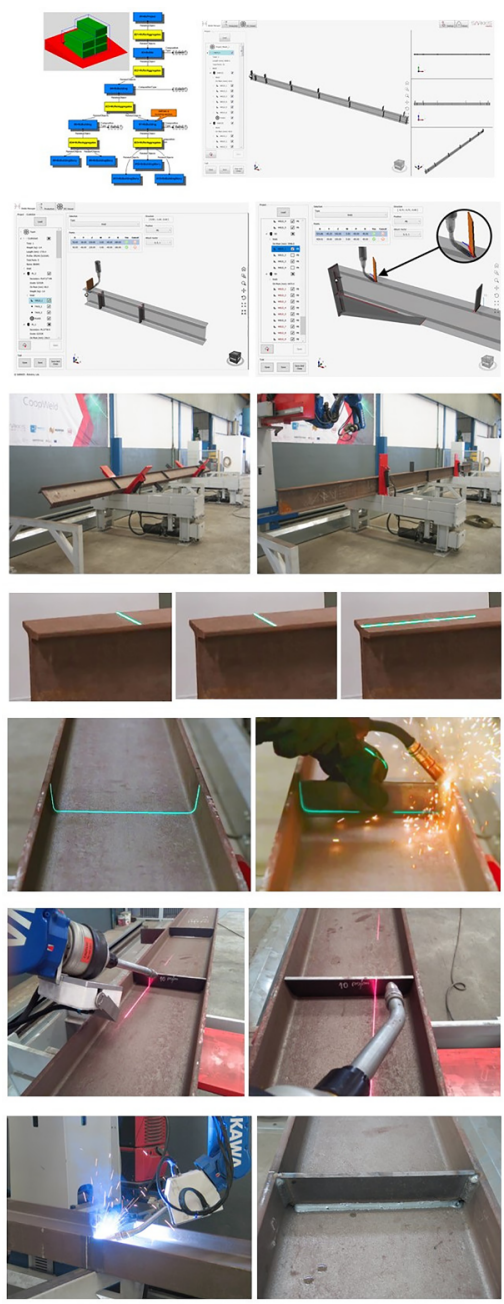

1 - Parsing of IFC file

- Extraction of all key data for the welding task

2 - Generation of tool approach vectors

- Estimation of the tool approach direction for the selected welds

\section{3 - Beam rotation}

- Rotation of the beam for improving robot reachability

\section{4 - Local calibration of galvanometer} scanner

- Estimation of beam pose in relation to laser projector

\section{5 - Spatial augmented reality}

- Projection of 3D information for helping the operator place and tack weld metal parts

\section{6 - Local calibration of beam} attachments

- Estimation of beam attachments location using laser triangulation

\section{7 - Production}

- Estimation of collision-free trajectories for the robot

- Generation and transmission of robot program

- Monitorization of robot production

Fig. 2. Overview of main processing stages of the CoopWeld cell.
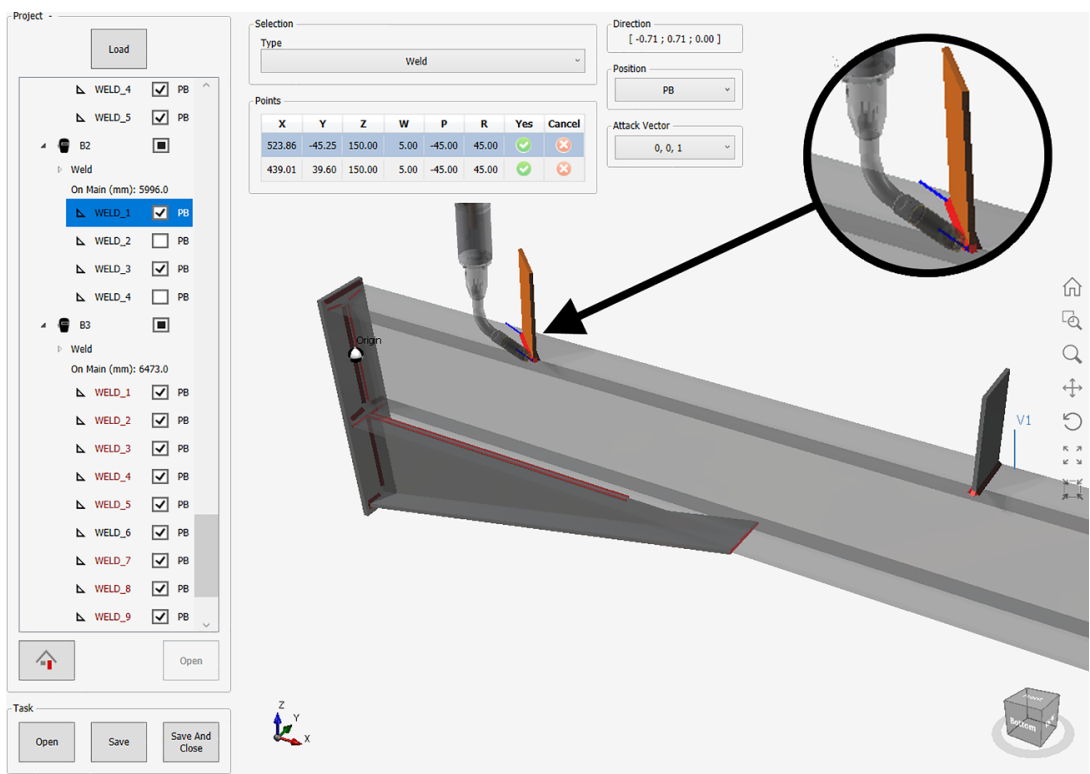

Fig. 3. MetroID BeamWeld Software. 


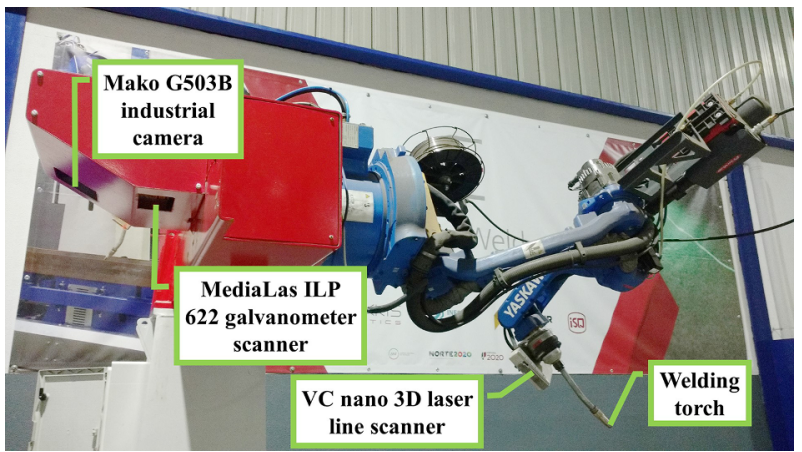

Fig. 4. SAR hardware (left) mounted on the moving track tower alongside the welding robot (right)

tolerances required by most welding factories (bellow $3 \mathrm{~mm}$ of error), the SAR system must be properly calibrated and it must also be able to detect where are the physical objects that will be used as a surface for projecting information. Moreover, the projected data must be clearly visible even in highly illuminated factories. With these requirements in mind, we chose laser galvanometer scanners [25] as our projection hardware (in particular, the MediaLas ILP 622) and developed the calibration tools, beam perception software and vector rendering pipeline for achieving a reliable and accurate SAR system for welding applications.

\subsubsection{Hardware Layout}

In our particular welding cell, the robotic arm is moving on a linear track and is attached to a tower for improving its reachability when welding on the top section of the beam. As such, for avoiding a dedicated support for the galvanometer scanner, we attached it on the opposite side of the robotic arm (as seen in Fig. 4) and at the minimum distance from the beam that would allow to project on our intended work area, which currently are beams with at most IPN $500^{1}$ dimensions that have a cross section of $500 \mathrm{~mm}$ in width and $185 \mathrm{~mm}$ in height.

By attaching the projector to a movable robot it makes the whole projection system more compact and more cost effective, since a single galvanometer scanner is enough for projecting marking information in our welding cell (which can handle beams with up to 12 meters in length). If we had chosen to attach several static projectors to the ceiling it would result in a more expensive and less accurate SAR system and it might also have occlusion problems due to the robot and its moving tower.

The hardware layout shown in Fig. 6 maximizes the precision of our SAR system because it uses the full field of view of the galvanometer scanner, allowing to place the projector as close as possible to the objects in which it will be projecting information (beam). This layout also makes SAR system more tolerant to slight errors in calibration (that introduce projection displacements that are linearly magnified as the projector becomes farther away from its projection surface). For example, assuming a vertically projected line on top of a horizontal plane, we can use trigonometry to find the spatial displacement on the horizontal plane that will occur when a rotation error is introduced by the overall calibration of the projection system (which includes the intrinsic and extrinsic calibration of the projector and camera along with the detection of where is the beam in relation to the projector). Namely, Eq. (1) allows to conclude that at $1 \mathrm{~m}$, a 0.1 degrees of rotation error will introduce a $1.75 \mathrm{~mm}$ of projection displacement while at $3 \mathrm{~m}$ the error would have increased 3 times to $5.25 \mathrm{~mm}$.

\footnotetext{
${ }^{1}$ https://www.cad-steel.eu/steel-sections/ipn-european-standard-beams
}

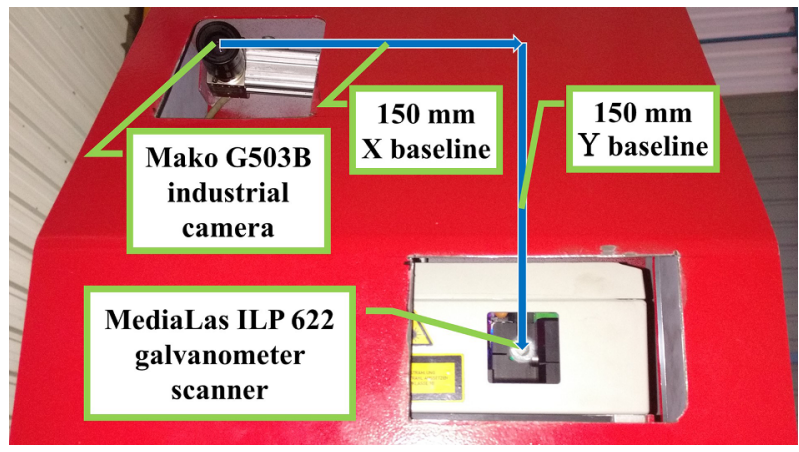

Fig. 5. Relative disposition between the camera (top left) and the galvanometer scanner (bottom right).

\section{HorizontalDisplacement $=$ Tangent $($ Rotation $) \times$ ProjectorHeight}

For performing laser triangulation using lines projected by the galvanometer scanner we also attached a 5 MP Mako G503B grayscale industrial camera with a $12 \mathrm{~mm}$ lens. We place it with a $\mathrm{X}$ and $\mathrm{Y}$ base line distance of around $150 \mathrm{~mm}$ for ensuring that we could generate accurate 3D measurements in our sensing volume (hardware layout shown in Figs. 5 and 6). The large baseline distance between the camera and projector is due to the extensive work area in which we need to perform measurements. Namely, the perception of beams with up to IPN 500 dimensions at a distance from the camera of around $1200 \mathrm{~mm}$ when they are in a $\mathrm{H}$ position and around $900 \mathrm{~mm}$ when they are positioned in a I configuration. Moreover, this large baseline was split into two axis components for ensuring that the vertical and horizontal projected light planes (used for detecting where is the beam in relation to the projector) did not came close to the camera optical axis (since this would cause severe loss of triangulation precision).

\subsubsection{Hardware Calibration and Beam Perception}

Projection mapping systems are able to seamlessly integrate into the

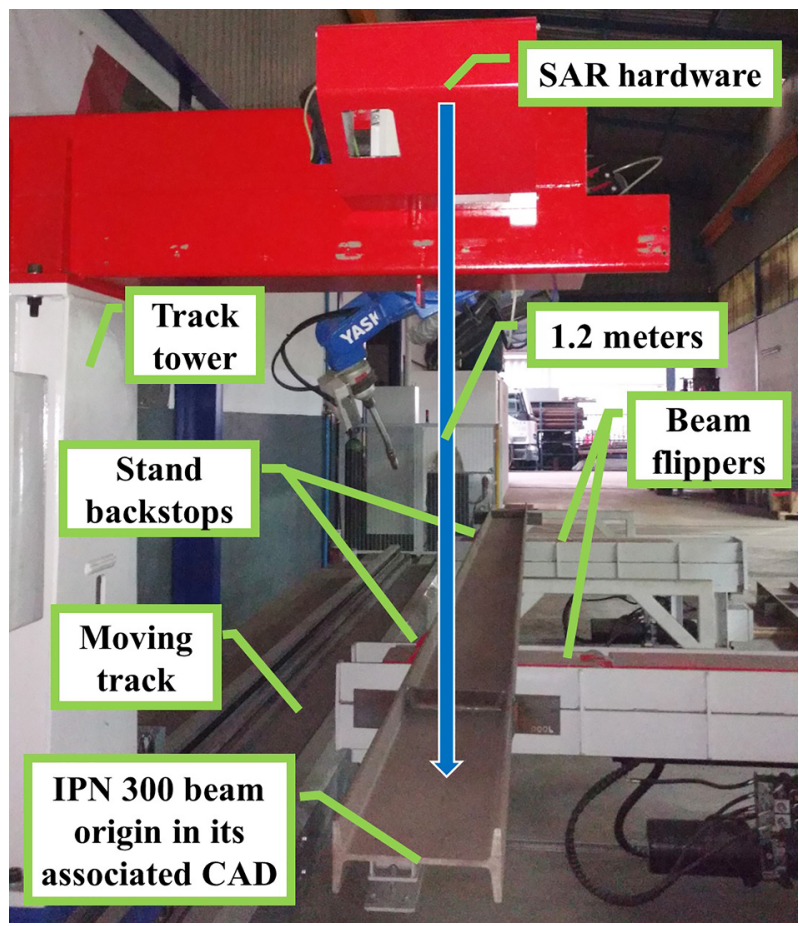

Fig. 6. Overview of the SAR hardware (galvanometer scanner and camera at the top of the image) mounted on a moving track and its projection target below (IPN 300 beam, laying on top of two beam flipping platforms). 

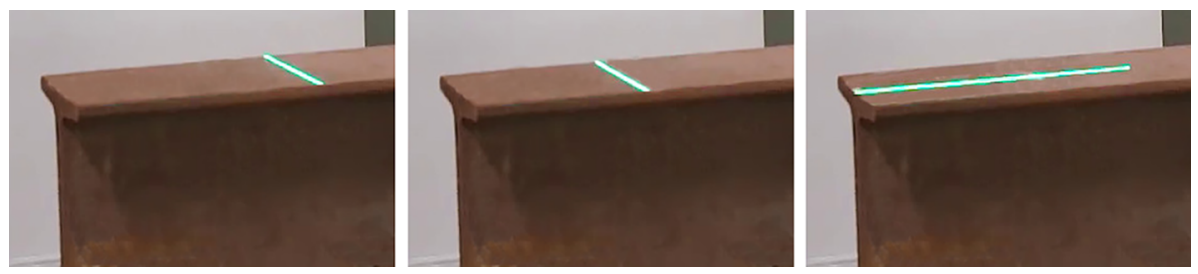

Fig. 7. Projection of two transversal lines (left and center) for estimating the beam direction and distance in relation to the projector and also a longitudinal line (right) for computing the beam start position along the moving track.

environment and provide immersive interfaces because the information is shown directly on top of the surfaces in which it is needed without requiring the operator to wear any extra devices. For achieving this level of accuracy the sensing and projection hardware must be properly calibrated $[26,27]$ and the workspace must be virtualized and maintained in a consistent state with the physical world.

For performing the 3D virtualization of the welding cell we used the Eyeshot CAD rendering engine ${ }^{2}$ and for ensuring proper simulation of the projector within the 3D environment we modeled the galvanometer scanner as a virtual pinhole camera [28] (which will be used for generating the $2 \mathrm{D}$ vector images required for projecting the HMI information).

For configuring the virtual pinhole camera model, we developed a calibration system for estimating the intrinsic and extrinsic parameters of both the projector and its attached camera. Moreover, we also developed a system for performing visual inspection of the calibration and perception modules for ensuring it is ready for production (example in Fig. 8).

For ensuring consistency between the virtual and physical world, we implemented a 3D beam perception module for updating the pose of the simulated camera within the Eyeshot virtual workspace. Our capability of using the galvanometer scanner hardware for both HMI projection and laser triangulation is critical for being able to estimate the beam position in relation to the projector with high precision (which is a requirement for proper projection mapping).

Given that the beam is placed on top of a rotating stand and is touching its 2 alignment backstops (shown on the left side of the beam in Fig. 6), our perception system only needs to correct for operator placement errors (along the track direction) and also beam bending problems. For dealing with the first issue we project a line along the beam longitudinal axis and estimate where is the start of the beam in relation to the projector and the moving track start position (this way we are able to accurately project information even if the camera is not seeing the beam start since we can rely on the high accuracy track encoder to update the projector position when the track moves). For computing the necessary corrections for dealing with the second problem we project two lines across the beam and estimate each line 3D centroid (on the beam top face) for computing the beam direction and also its height in relation to the projector (example of the projection lines on top of a IPN 300 beam in a I configuration shown in Fig. 7). We rely on the beam center (origin of the CAD coordinate system shown in Fig. 6) as a common reference frame between the virtual and physical beams because its width and height may vary a few millimeters due to manufacturing problems. As such, for our welding use cases, aligning the virtual and physical beams centers for evenly distributing the manufacturing error on both sides of the beam seems to be the best approach to deal with these issues.

Since our welding cell can operate on beams with up to $12 \mathrm{~m}$ in length, we needed to rely on the tower linear track encoder for estimating how much did the projector move in relation to the beam beginning. As such, when a new beam is placed within the welding cell or it is rotated by the stand, we move the tower to the beam beginning and

\footnotetext{
${ }^{2}$ https://www.devdept.com
}

correct its displacement in relation to the projector. Then we can trust on the high accuracy linear track encoder to update the projector pose as long as the beam is not moved. On the other hand, since the beam bending might be irregular, when the tower moves to a new welding position we perform a local calibration (using the approach that relies on the two transversal lines discussed earlier) in which we update the virtual camera pose for mitigating the beam bending issues.

\subsubsection{Generation of HMI Information from IFC}

The Industry Foundation Classes (IFC) is a neutral and open file format that can be used for exchanging BIM specifications between designers and constructors. It provides in a single file the CAD data and the meta-information (such as their spatial disposition and welding sections) required to produce structural steel assemblies. Within our particular welding cell configuration we start by loading the main structural beam from the IFC file and then incrementally add the HMI information and CAD models of the parts that must be welded later on (such as plates on the beam flange, web or end sections for attaching the beam to other structures or reinforce its connections). Our HMI system provides two different and complementary ways for conveying to the operator the assembly and welding information that was extracted from the IFC file. The first is through a large tactile monitor in which the fully assembled parts are shown in several 3D views along with instructions and controls for the operations that are required (examples presented in Figs. 9 and 11). The second interface relies on the IFC system for projecting alignment shapes directly into the beam surface for helping the operator place and tack weld the beam attachment structures (examples shown in Figs. 10 and 12). This alignment information is automatically generated by computing the intersection lines between the CAD models of the beam and its attachments followed by a post-processing stage for simplifying and optimizing the lines for reducing projection flickering.

For validating and also evaluate the accuracy of the proposed approaches for calibrating the SAR hardware and generate its information from BIM, we performed several tests in which marking information

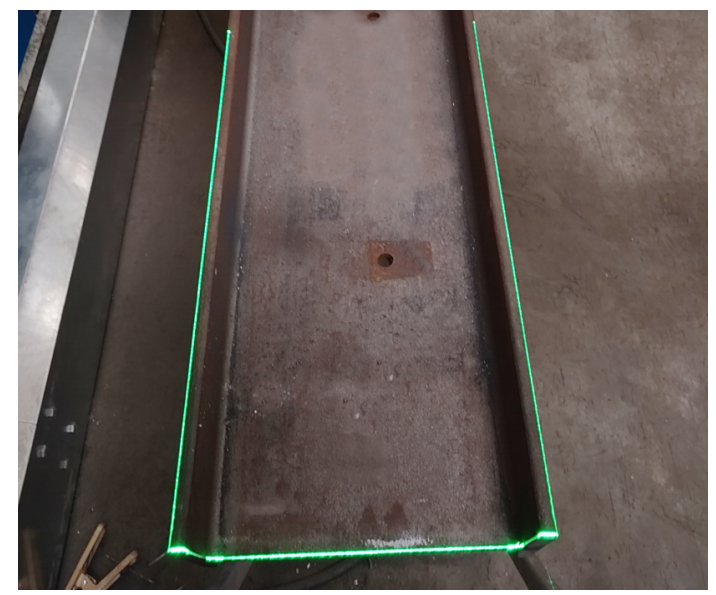

Fig. 8. Projection of the beam outline (in green) for performing a visual inspection of the SAR hardware calibration and the beam perception modules. 


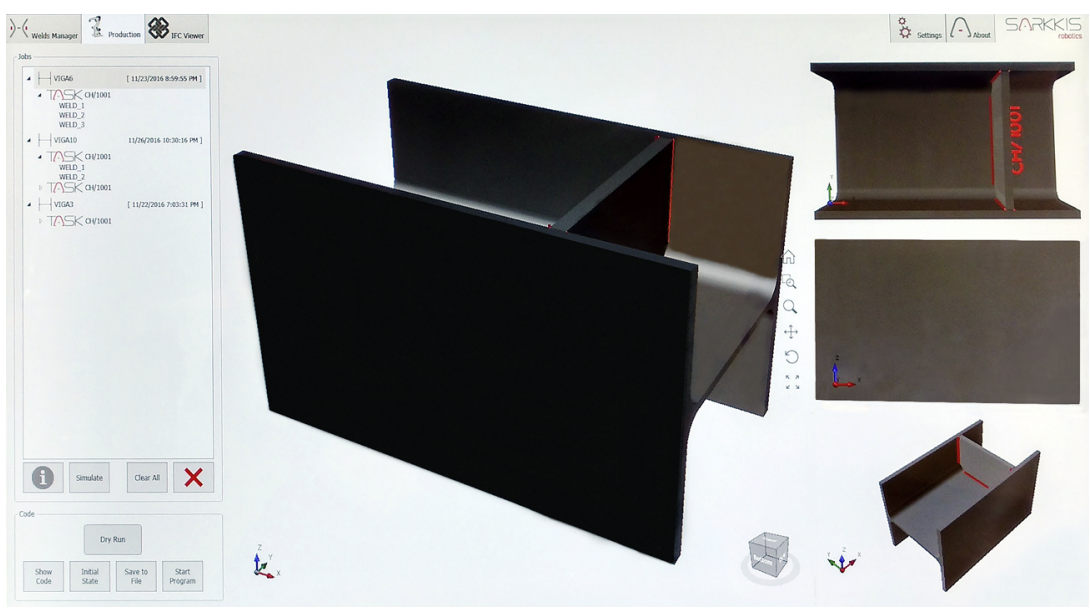

Fig. 9. Tactile user interface for a small demonstration beam.
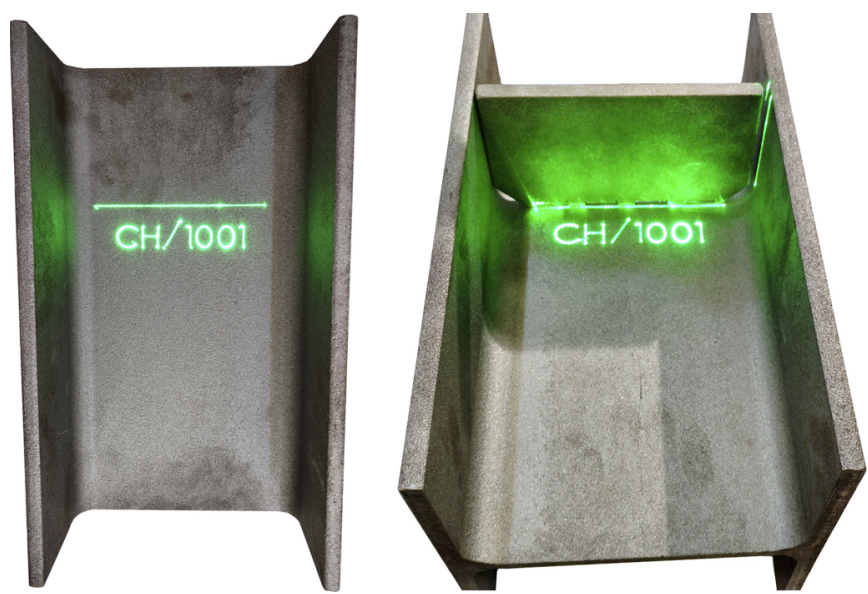

Fig. 10. Projection of alignment information for the placement of the beam attachment shown in Fig. 9.

was projected for informing the operator where he should place beam attachments (example in Fig. 12). Our experimental evaluation showed that the SAR system was able to achieve a projection error below $3 \mathrm{~mm}$, making it suitable for the proposed welding applications and our end users.

\subsection{High Precision Laser Based Localization System for Robotic Welding}

The placement phase of the metal structure in the robot's workstation is usually performed manually using an industrial crane due to the weight of the structure. However, given its inherent inaccurate placement, it does not meet the positioning requirements for the welding operations to be performed automatically by an industrial robot.

In the CoopWeld robotic cell (shown in in Figs. 6 and 20) the number of degrees of freedom in which the beam can be moved relative to the robot is minimized because we rely on a flipping stand (shown in Fig. 13) that includes side stops (displayed in Fig. 6). These flippers provide enough flexibility to adjust the beam position and rotation in relation to the robotic structure, despite the position the beam is placed at the start. As such, most of the error introduced by the operator is along the beam longitudinal axis. In spite of this reduction on the problem complexity, the presence of a machine vision system for the dimensional validation of the beam and its localization respectively to the robot base frame is still mandatory. This system allows to perform the adjustment of the beam reference frame programmed in the robot, over which the robot's welding trajectories will be generated later on.

Moreover, the components to be welded in the robotic cell will also be inserted and tack welded manually by a human operator. The operator at this stage will have a valuable support provided by the projection mapping system, which will indicate the correct location for that placement based on the information taken from the CAD / IFC files.

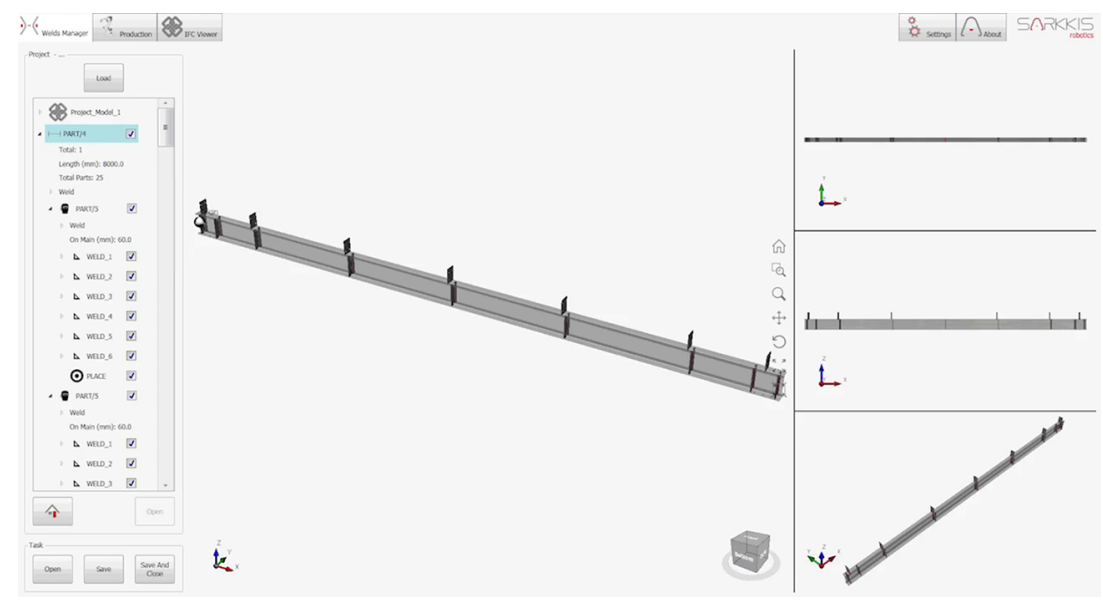

Fig. 11. Tactile user interface for the production of a structural beam. 


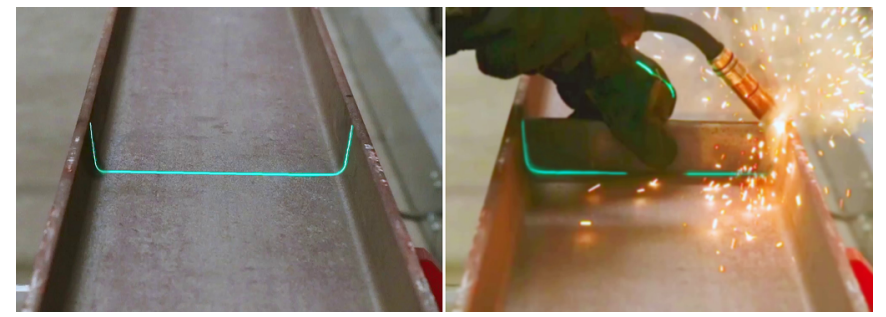

Fig. 12. Projection of alignment information for one of the beam attachments shown in Fig. 11.

However, as it is a manual operation, the placement of the components will have some positional error when compared to the theoretical location indicated by the projection system. This difference has direct implications in the trajectory to be performed by the industrial robot and will affect the quality of the finished product. Due to these characteristics, it is also necessary to provide the robotic system with a vision system capable of detecting the joint to be welded, and in particular to validate the positioning of the part inserted by the human operator.

To circumvent these problems, a machine vision system was developed that relied on a 2D laser scanner (VC Nano 3D) that was attached alongside the robot welding torch shown in Fig. 14. In this sense, the first effort went into the calibration of the laser scanner in the industrial robot Tool Center Point (TCP).

\subsubsection{Laser Scanner TCP Calibration}

For the calibration of line laser scanner sensor in the TCP of the industrial robot it was used the methodology presented in [29,30]. This laser calibration heuristic runs in two distinct steps and it is based on the scanning of a physical sphere with known diameter. First, using different pairs of lasers scans over the physical sphere, each with different tool $l_{0}$ orientations, it is calculated the rotation matrix between the robot tool $l_{0}$ reference and the laser scanner reference frames. Then, keeping the robot orientation unchanged to scan a complete sphere surface, the translation vector is calculated between the same frames, using as input the rotation matrix computed in the first step. For more detail on the followed methodology please refer to the works described in $[29,30]$.

\subsubsection{Laser Vision System for Beam Localization}

Due to the manufacturing tolerances of the steel structures, some adjustments must be made to the robot theoretical trajectories. Moreover, the detection of the beam origin is mandatory to ensure a

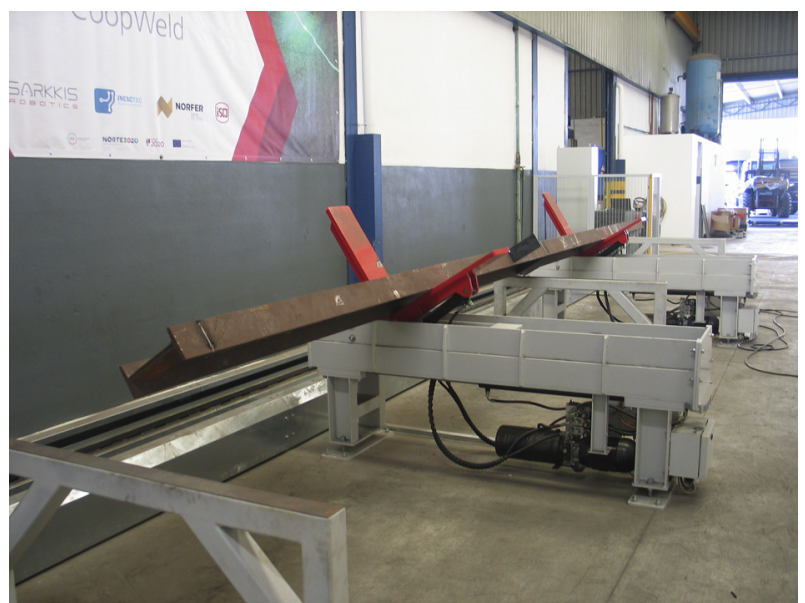

Fig. 13. Flipping stand.

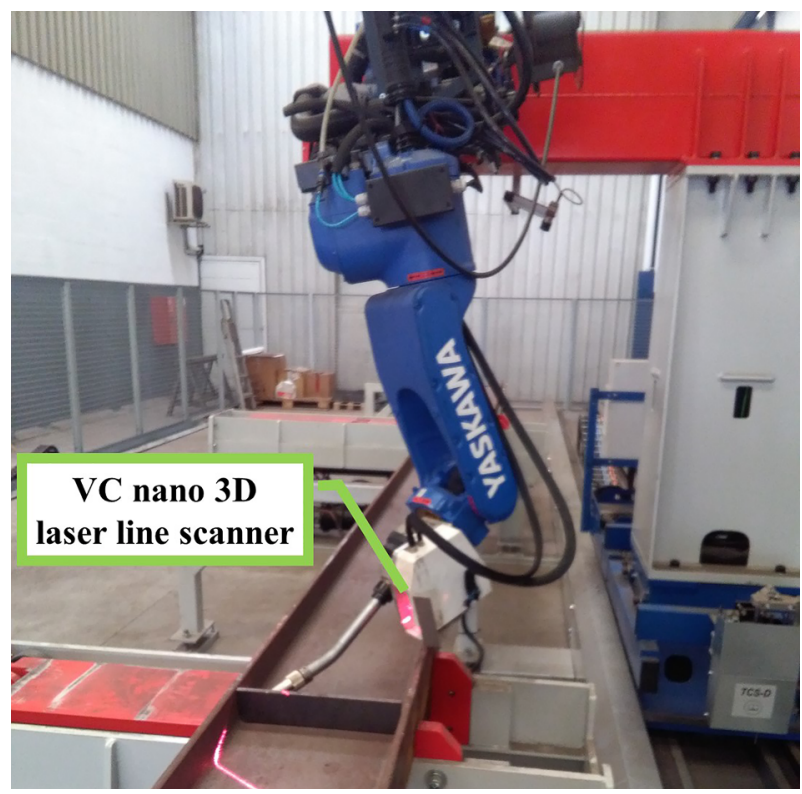

Fig. 14. Hardware layout for the high precision laser based localization system for robotic welding.

correct work process. The achievement of this measures is made using a base frame in the robot (normally located in a fixed position). Then a calibration process is run to detect some of the edges of the beam, as shown in Fig. 15. With the information obtained in the calibration process it is possible to adapt the welds to the real parts dimensions as well as ensure a correct execution of the planned trajectories.

\subsubsection{Laser Vision System for Simultaneously Localize and Validate} Manually Inserted Parts Position

Considering the problem context presented earlier, it was also developed a system capable of detecting the joints of the parts that need to be seam welded by the robot (since the operator may have introduced positioning errors when it tack weld them). As such, to validate the position of the component in the steel structure, the position of the joint was considered as an initial good estimate. Later on, by comparing the theoretical value (obtained in the virtual beam loaded from the IFC file) with the sensor data acquired with the laser scanner (example in Figs. 16 and 17), it is possible to validate its position and directly compensate the trajectory of the industrial robot.

For welding, this detection should ideally be performed on the first point of the welding path of the industrial robot, where the correction would then be applied. This is due to the fact that the component to be welded might have small deformations which would be tolerated by the industrial COMARC joint follow-up system installed in the CoopWeld cell. However, given the geometric dimensions of the laser scanner sensor and its scope, after some preliminary tests executed in a industrial environment, it was verified that there were occasions where it would be complicated to ensure this requirement. In particular, with regard to the guarantee of non-collision between the torch and the beam.

To overcome this difficulty, a new heuristic was designed, in which instead of extracting only one point of the joint to be welded, at least three points are detected (as depicted in Fig. 18). The offsets between these three points are automatically computed based on the the theoretical length of the part to be welded. Later on, using a linear regression algorithm it is possible to compute the joint vector equation of the line and then project the theoretical welding trajectory point of the industrial robot for detecting the position of the joint to be welded. 


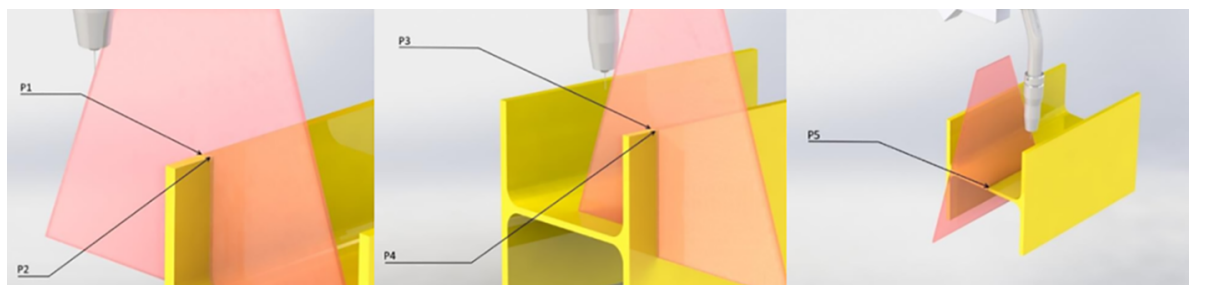

Fig. 15. Beam localization procedure.

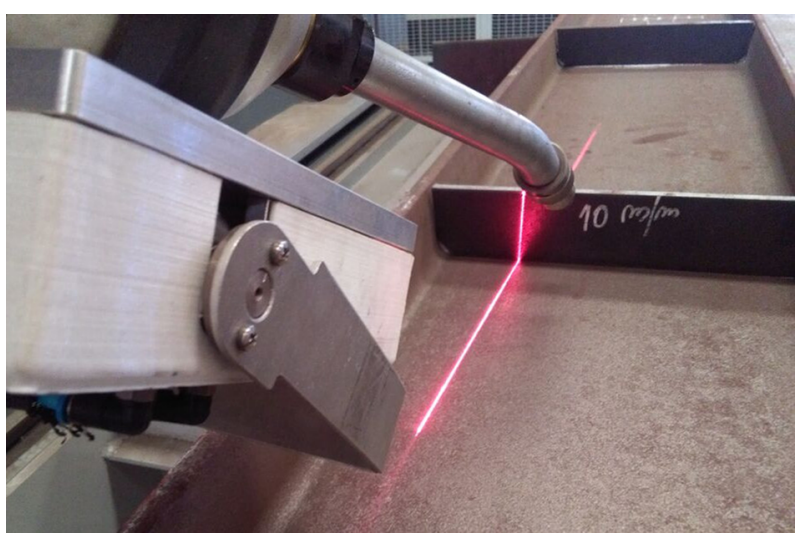

Fig. 16. Detection of the joint to be welded using the 2D laser scanner.
TS 15066:2016 for collaborative robots, an add-in to the robot was inserted to fulfil the requirements of hand-guiding robotic system. As stated in Fig. 19, it is possible to identify two directional buttons and one emergency stop button that allows the user to move the robotic system track and consequently acquire information for each part of the element to be produced.

The end guiding feature allows the user to control the robotic system state in order to get a better grasp on the task and the placement that will be require next. These proposed features provide a collaborative work cell between the robotic system and an human operator.

The optimal TCP pose of the welding torch is reached based on a multi-parameter cost function that tries to minimize velocities, changes of configuration and robot / track effort, while maximizing the system range for future tasks. Once this pose is optimized, then a probabilistic path planning algorithm is used to compute a collision-free path that guides the robotic system from a given pose to the goal destination.

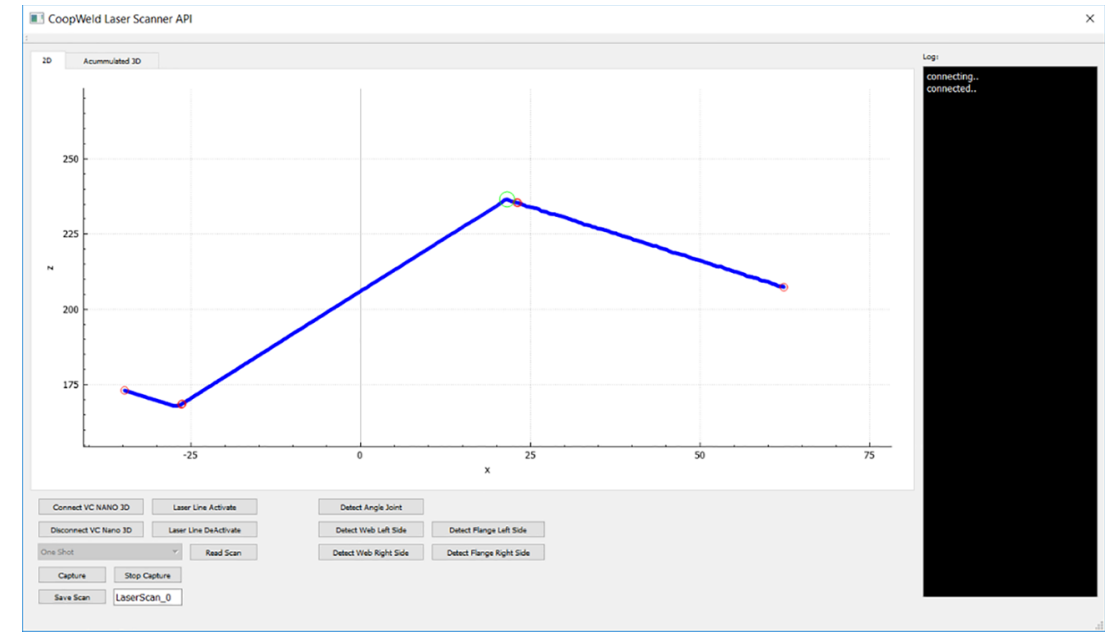

Fig. 17. Result of laser scanning on a joint formed by the intersection of a reinforcement cutter and the core of the beam.

\subsection{Robotic System Control and Trajectories Optimization}

In order to optimally control the robot system and its trajectories, a heuristic-based methodology using a genetic algorithm was implemented. This methodology uses the information obtained from the Computer Aided Manufacturing Software of MetroID BeamWeld and generates the optimal configuration for the robot, track and external components (in this case the flippers).

The robot platform mounted on the moving track carries a portable welding machine that is available to the human operator. The concept behind this work cell considers two stages. The first is the placing and tack weld of a given part by the operator while the second is the automatic seam welding by the robotic system. To accomplish the first task, the projection mapping system provides the user 3D marking information that is projected directly into the beam for speed up the assembly of beam attachments. This allows the human operator to perform his task in a more efficient and precise way, placing and tack welding the parts in the proper location. Then, following the norm ISO/

\section{Robotic System for Welding}

The current work is focused on the design of a collaborative robotic cell for structural steel beam welding. The robotic system is composed by a welding robot attached to a mobile track. In order to perform the rotation handling of the beam, there are also two flippers that can move and rotate the beam. Furthermore, all the security requirements are complied by using precise light barriers, emergency buttons and force sensors.

The CoopWeld ${ }^{3}$ work cell is presented in Fig. 20 and a demonstration video of its operation is available at ${ }^{4}$.

This work cell was designed based on the principals of collaborative robots as its components were adapted to better integrate with a human operator. Furthermore, the design was also an outcome of a genetic

\footnotetext{
${ }^{3}$ https://www.coopweld.com

${ }^{4}$ https://youtu.be/3L0JBA9ozFA
} 

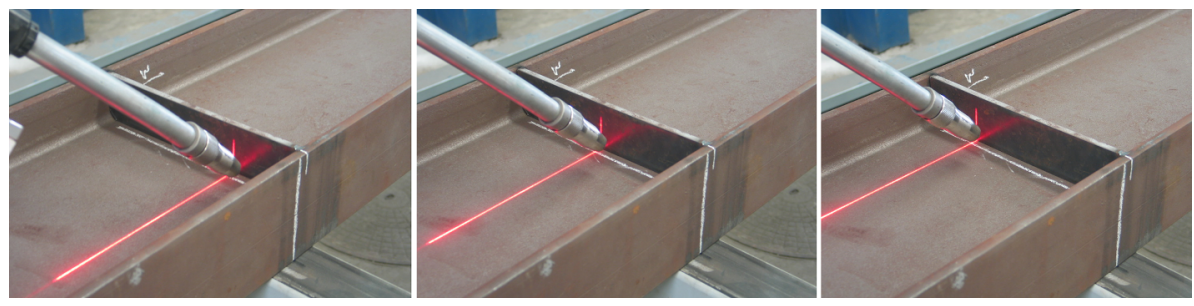

Fig. 18. Illustration of the three point approach for the detection of the welding joint.

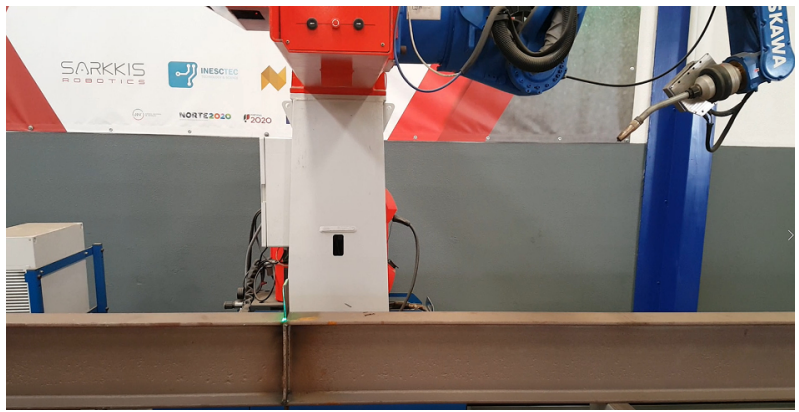

Fig. 19. Hand Guiding Example.

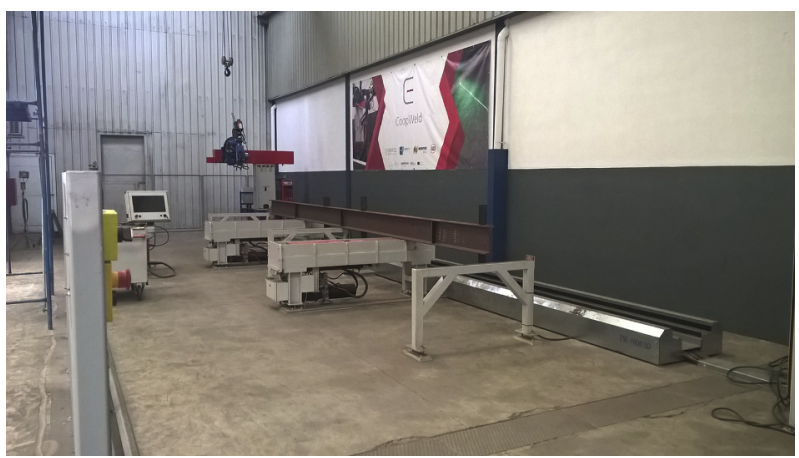

Fig. 20. CoopWeld: Beam Welding Work Cell.

algorithm for work cell design and job validation. In this regard, an optimization technique was used to ensure the robot position that promotes the robotic challenge of the cell as well as the supporting structure.

\subsection{Welding Work Flow}

The collaboration between the human operator and the robotic system is highly enhanced in this work cell regarding the preparation stage of the tasks. Furthermore, this welding cell was designed and developed to be flexible and extensible in order to allow manual operation, semi-automatic collaboration and fully automated welding. Therefore, each job to be completed using this system can be divided in a set of steps. First there is the need to identify the BIM file that will carry the required information for the job. Then an analysis on this file is performed and crucial welding operations are identified and converted into tasks. These tasks then demand cooperation between the robotic system and a human operator. While the first is responsible for automated welding tasks (final stage of the job), it is also crucial for projection mapping tasks for helping the operator during its tack weld operations. Here we need to have a close relation between both ends of this collaborative system, hence the developed HMI is relevant to easily allow the communication between them and the separate portable welding device allows the job completion in a efficient way.

The optimization of poses and the insertion of a collision-free path planner gives the ability of welding always in horizontal vertical position (PB) which contributes for a cleaner weld (an example of the final result is presented in Fig. 21).

\subsection{Robot Welding vs Traditional Welding}

Focusing on usual beam welding tasks it is possible to perform a comparison between robot efficiency when compared to traditional welding operators. In order to do so, we've subjected both traditional operators and the designed robotic system to perform the complete welding procedure on some beams (Fig. 22 shows the test scenarios).

For the first test case, we have a total assembly weight of $383.26 \mathrm{~kg}$. This is a simpler challenge as the total weld length is $2.56 \mathrm{~m}$ with a throat size of $6 \mathrm{~mm}$. The complete deposited material in distance is 4.90 $\mathrm{m}$, corresponding to a weight of $1.11 \mathrm{~kg}$. For the second test case, we have a total assembly weight of $1052.78 \mathrm{~kg}$. The total weld length is $12.72 \mathrm{~m}$ with mixed throats of $6 \mathrm{~mm}$ and $8 \mathrm{~mm}$. The deposited quantity is of $24.55 \mathrm{~m}$ in a total of $5.24 \mathrm{~kg}$ of solder.

Regarding the welding quality, both test cases present similar results. The weld parameters are also consistent and similar for both the operator and the robotic system. The only key advantage during the welding procedure is related to system repeatability and endurance. Despite the fact that real welding time is similar, the robot can ensure non-stop operation while the operator fatigue and motion along the beam tends to slow the process and deteriorate the overall welding quality.

The most contrasting factor is the total operation time. The proposed system provides enough information and automation to eliminate placing errors (that require future adjustment) and the re-position of components such as beams or secondary parts. Once again, referring to the presented test cases, the first one corresponds to a robot welding time of 18 minutes and 11 seconds in a 49 minutes and 58 seconds overall cycle time. The traditional operator here achieves welding times bounded between 18 and 21 minutes and a total cycle time around 132 minutes. For the second example, we have a robotic welding time of 85 minutes and 2 seconds on a total cycle time to 116 minutes and 49 seconds versus a operator welding time between 90 and 100 minutes and a total cycle time around 310 minutes.

Analyzing the results achieved by proposed CoopWeld work cell, it is notorious the improvement that it can give in the overall production. Moreover, the efficiency of the machine and its ability to operate continuously and with high repeatability are the greatest benefits when compared to traditional welding using human operators.

\section{Conclusions and Future Work}

Throughout this article we presented a solution regarding robotic welding enriched by the concept of human and robot collaboration for providing a wide range of flexibility for being applied to other robotic systems with the same purpose. This solution complies with the safety requirements for collaboration between robotic machinery and humans, having already been applied in an industrial use case for structural steel beam welding.

High redundancy robotic systems commonly lack in flexibility regarding interoperability between human and robotic operators. Hence, the output of this project is clearly valuable as it presents a 

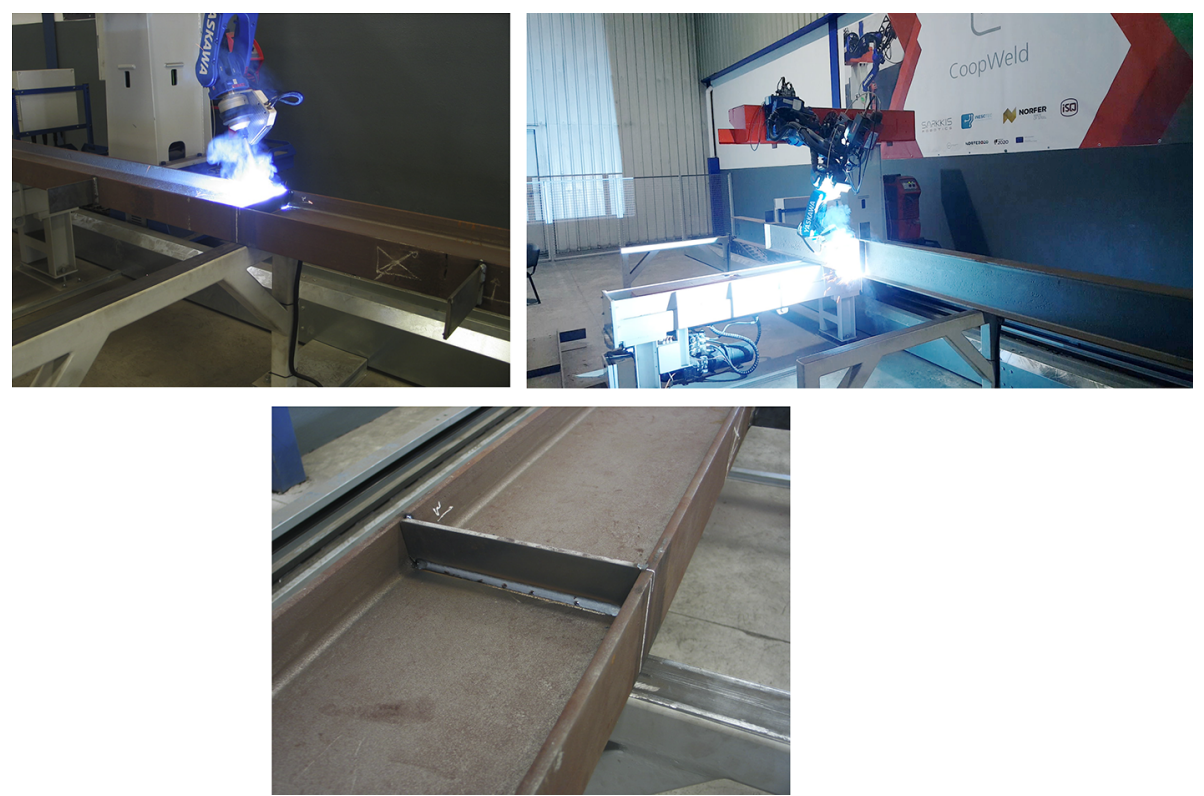

Fig. 21. Seam weld of a beam attachment performed by the CoopWeld work cell.

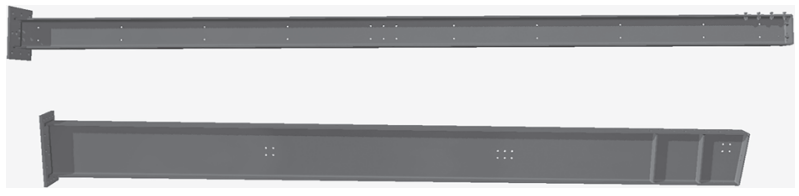

Fig. 22. Test scenarios for two beams.

collaborative solution based on key developments for the robotic world. The standardization of information flow via BIM, and in particular via IFC files, enables the traceability of the process and a more efficient sequencing of tasks to complete such process.

The SAR system using projection mapping techniques allowed to maximize the usefulness of the information present in the IFC file format by showing spatial alignment markings directly into the environment. Besides improving the overall productivity of the operator, it also contributes to the drastic reduction of manufacturing mistakes since the operators no longer needs to use error prune measurement tools. Moreover, by showing the specifications on demand, this HMI system also removes from the operator the burden of constantly checking the architect design, minimizing interpretation issues.

The hand guiding allows the human operator to easily control the robotic system in order to identify the parts placements. Then the realtime adjustments computed from laser corrections allow the robotic system to automatically adapt the information retrieved from the BIM. Moreover, this particular welding cell takes advantage of the usage of multiple optimization algorithms regarding robot control and operation management. The usage of an optimal positioning planner allows to perform welding operations in horizontal vertical position (PB). This contribution has been highly valued within the industrial environment due to its effectiveness in producing cleaner results and can be extended to any other system or operation. Future perspectives for this kind of application are mainly directed to its scalability, namely its deployment in work cells with higher complexity that may include higher degrees of freedom and also dynamic collisions with the environment.

In conclusion, the collaborative robotic welding system presented maximizes the usefulness of BIM for producing optimized information flow and is able to perform complex and optimal welding tasks alongside human operators.

\section{Acknowledgments}

This work is financed by the ERDF - European Regional Development Fund through the Norte Portugal Regional Operational Programme (NORTE 2020), and through the Portuguese National Innovation Agency (ANI) as a part of project CoopWeld | NORTE-010247-FEDER-006438.

\section{References}

[1] buildingSMART, buildingSMART International Ltd, On-line: http://www.buildingsmart-tech.org/ (Accessed: 09/03/2019).

[2] M. Hvilshøj, S. Bøgh, "Little helper" - An autonomous industrial mobile manipulator concept, Int. J. Adv. Robot. Syst. 8 (2) (2011), https://doi.org/10.5772/10579.

[3] J.-h. Kim, Automated medicine storage and medicine introduction/discharge management system, Oct 9, 2012, 8,281,553 United States Patent.

[4] P. Westhead, M. Wright, D. Ucbasaran, The internationalization of new and small firms: A resource-based view, J. Bus. Ventur. 16 (4) (2001) 333-358, https://doi. org/10.1016/s0883-9026(99)00063-4.

[5] E. Holtzhauer, H. Saal, Product modelling in the steel construction domain, (Dec 2004), https://doi.org/10.25643/bauhaus-universitaet.241.

[6] I. JIvkov, Is a Robot in your Future? Modern Steel Construction 51.5, 2011, pp. 58-59.

[7] G. van Nederveen, F. Tolman, Modelling multiple views on buildings, Autom. Constr. 1 (3) (1992) 215-224, https://doi.org/10.1016/0926-5805(92)90014-B.

[8] B. Succar, Building information modelling framework: A research and delivery foundation for industry stakeholders, Autom. Constr. 18 (3) (2009) 357-375, https://doi.org/10.1016/j.autcon.2008.10.003 cited By 442.

[9] S. Chen, Y. Li, N. Kwok, Active vision in robotic systems: A survey of recent developments, Int. J. Robot. Res. 30 (11) (2011) 1343-1377, https://doi.org/10. $1177 / 0278364911410755$.

[10] ISO, ISO/TS 15066:2016 -Robots and robotic devices - Collaborative robots, Online: https://www.iso.org/standard/62996.html (Accessed: 09/03/2019).

[11] O. Bimber, R. Raskar, Spatial Augmented Reality: Merging Real and Virtual Worlds, A. K. Peters, Ltd., Natick, MA, USA, 2005.

[12] A. Doshi, R.T. Smith, B.H. Thomas, C. Bouras, Use of projector based augmented reality to improve manual spot-welding precision and accuracy for automotive manufacturing, Int. J. Adv. Manuf. Technol. 89 (5) (2017) 1279-1293, https://doi. org/10.1007/s00170-016-9164-5.

[13] M.L. Yuan, S.K. Ong, A.Y.C. Nee, Assembly Guidance in Augmented Reality Environments Using a Virtual Interactive Tool, Innovation in Manufacturing Systems and Technology, 2005, pp. 1745-1767.

[14] A. Nee, S. Ong, G. Chryssolouris, D. Mourtzis, Augmented reality applications in design and manufacturing, CIRP Ann. 61 (2) (2012) 657-679, https://doi.org/10. 1016/j.cirp.2012.05.010.

[15] J. Zhou, I. Lee, B. Thomas, R. Menassa, A. Farrant, A. Sansome, Applying Spatial Augmented Reality to Facilitate In-situ Support for Automotive Spot Welding 
Inspection, Proceedings of the 10th International Conference on Virtual Reality Continuum and Its Applications in Industry, ACM, 2011, pp. 195-200, , https://doi. org/10.1145/2087756.2087784.

[16] S. Makris, P. Karagiannis, S. Koukas, A.-S. Matthaiakis, Augmented reality system for operator support in human-robot collaborative assembly, CIRP Ann. 65 (1) (2016) 61-64, https://doi.org/10.1016/j.cirp.2016.04.038.

[17] G. Michalos, N. Kousi, P. Karagiannis, C. Gkournelos, K. Dimoulas, S. Koukas, K. Mparis, A. Papavasileiou, S. Makris, Seamless human robot collaborative assembly - An automotive case study, Mechatronics 55 (2018) 194-211, https://doi. org/10.1016/j.mechatronics.2018.08.006.

[18] S. Makris, G. Pintzos, L. Rentzos, G. Chryssolouris, Assembly support using AR technology based on automatic sequence generation, CIRP Ann. 62 (1) (2013) 9-12, https://doi.org/10.1016/j.cirp.2013.03.095.

[19] A.E. Uva, M. Gattullo, V.M. Manghisi, D. Spagnulo, G.L. Cascella, M. Fiorentino, Evaluating the effectiveness of spatial augmented reality in smart manufacturing: a solution for manual working stations, Int. J. Adv. Manuf. Technol. 94 (1) (2018) 509-521, https://doi.org/10.1007/s00170-017-0846-4.

[20] G.F. Barbosa, J. de Carvalho, C.H.P. de Souza, Deployment of a laser projection solution for stripes plotting based on Six Sigma DMAIC methodology applied to aircraft painting shop, Prod. Manuf. Res. 2 (1) (2014) 697-711, https://doi.org/10. 1080/21693277.2014.943432.

[21] I. Iglesias, M. Sebastián, J. Ares, Overview of the State of Robotic Machining: Current Situation and Future Potential, Procedia Eng. 132 (2015) 911-917, https:// doi.org/10.1016/j.proeng.2015.12.577 mESIC Manufacturing Engineering Society
International Conference 2015..

[22] Y. Xu, N. Lv, G. Fang, S. Du, W. Zhao, Z. Ye, S. Chen, Welding seam tracking in robotic gas metal arc welding, J. Mater. Process. Technol. 248 (2017) 18-30, https://doi.org/10.1016/j.jmatprotec.2017.04.025.

[23] OpenBIM, OpenBIM, On-line: http://www.openbim.org/ (Accessed: 09/03/2019).

[24] I. Wiki, IFC Wiki, On-line: http://www.ifcwiki.org/ (Accessed: 09/03/2019).

[25] W. Benner, R. Smith, Laser Scanners: How They Work, and How They Can Work for Your Product: Technologies and Applications, Pangolin, 2016.

[26] Z. Zhang, A flexible new technique for camera calibration, IEEE Trans. Pattern Anal. Mach. Intell. 22 (11) (2000) 1330-1334, https://doi.org/10.1109/34.888718.

[27] A. Manakov, H.-P. Seidel, I. Ihrke, A Mathematical Model and Calibration Procedure for Galvanometric Laser Scanning Systems, in: P. Eisert, J. Hornegger, K. Polthier (Eds.), 16th International Workshop on Vision, Modeling and Visualization, Eurographics Association, Berlin, Germany, 2011, pp. 207-214.

[28] R. Hartley, A. Zisserman, Multiple View Geometry in Computer Vision, 2nd edition, Cambridge University Press, 2003.

[29] J. Li, J. Zhu, Y. Guo, X. Lin, K. Duan, Y. Wang, Q. Tang, Calibration of a portable laser 3-D scanner used by a robot and its use in measurement, Opt. Eng. 47 (2008) 47-48, https://doi.org/10.1117/1.2829766.

[30] J. Li, M. Chen, X. Jin, Y. Chen, Z. Dai, Z. Ou, Q. Tang, Calibration of a multiple axes 3-D laser scanning system consisting of robot, portable laser scanner and turntable, Optik - Int. J. Light Electron Opt. 122 (4) (2011) 324-329, https://doi.org/10 1016/j.ijleo.2010.02.014 